\title{
Serum concentrations and peripheral secretion of the beta chemokines monocyte chemoattractant protein 1 and macrophage inflammatory protein $1 \alpha$ in alcoholic liver disease
}

\author{
N C Fisher, D A H Neil, A Williams, D H Adams
}

\begin{abstract}
Background-Alcoholic liver disease is associated with increased hepatic expression of monocyte chemoattractant protein 1 (MCP-1) and macrophage inflammatory protein $1 \alpha(\mathrm{MIP}-1 \alpha)$.

Aims-To determine whether concentrations of chemokines in the peripheral circulation reflect disease activity, and whether chemokine secretion is restricted to the liver or is part of a systemic inflammatory response in alcoholic liver disease. Patients-Fifty one patients with alcoholic liver disease and 12 healthy controls.

Methods-Peripheral vein (and hepatic vein in patients undergoing transjugular liver biopsy) chemokine concentrations were measured by ELISA. Chemokine secretion and transcription in isolated peripheral mononuclear cells were assessed using ELISA and in situ hybridisation in patients with severe alcoholic hepatitis.
\end{abstract}

Results-Serum MCP-1 concentrations were higher in alcoholic hepatitis compared with cirrhosis or healthy controls. MIP-1 $\alpha$ concentrations were below the assay sensitivity in most patients. Serum MCP-1 concentrations correlated significantly with serum aspartate aminotransferase and creatinine. In severe alcoholic hepatitis, MCP-1 concentrations were higher in hepatic compared with peripheral veins; in mild alcoholic hepatitis there was no difference. Mononuclear cell secretion of both MCP-1 and MIP-1 $\alpha$ was higher in severe alcoholic hepatitis compared with healthy controls, and chemokine mRNA was identified in monocytes. Conclusions-Serum MCP-1 concentrations are raised in alcoholic liver disease and reflect severity of hepatic inflammation. Monocyte secretion of both MCP-1 and MIP- $1 \alpha$ is increased in severe alcoholic hepatitis. Both intrahepatic sources and peripheral mononuclear cells contribute to the raised serum MCP-1 concentrations.

(Gut 1999;45:416-420)

Keywords: alcoholic liver disease; chemokines; monocyte chemoattractant protein 1 ; macrophage inflammatory protein $1 \alpha$

Alcoholic hepatitis is characterised by an intense leucocytic infiltration of the liver combined with hepatocyte damage and a variable degree of fibrosis. ${ }^{1}$ While the key hepatotoxin underlying alcoholic liver disease (ALD) is self evident, the cascade of immunological events leading to inflammatory damage is complex and the variation in inflammatory response between individuals consuming similar amounts of alcohol is not understood. ${ }^{2}$ We and others have previously shown that the expression of chemokines, a subgroup of cytokines with chemoattractant activity, correlates with clinical severity and inflammatory activity within the liver in alcoholic and other inflammatory liver diseases. ${ }^{3-7}$ The prinicipal role of chemokines is in the recruitment of leucocytes to sites of inflammation and they play a vital role in a variety of infective and inflammatory diseases. ${ }^{8}{ }^{9}$ Chemokines can be subdivided according to their structure into subgroups, of which the largest are the CXC, or alpha, and CC, or beta groups defined by the presence or absence respectively of an additional amino acid ("X") between the first two cysteine residues in a conserved four cysteine motif. ${ }^{9}$ The alpha chemokines are further subdivided according to the presence or absence of a glutamine-leucine-arginine (ELR) amino acid sequence near the active terminal; those possessing this sequence are potent chemoattractants for neutrophils while those that do not are chemotactic for lymphocytes. Interleukin 8 (IL-8) possesses an ELR amino acid sequence and is the prototypic alpha chemokine, being exclusively chemotactic for neutrophils. The beta chemokines are exclusively chemotactic for mononuclear cells; the prototypes of this group are monocyte chemoattractant protein 1 (MCP-1) and macrophage inflammatory protein $1 \alpha(\mathrm{MIP}-1 \alpha)$, both of which are monocyte and lymphocyte chemoattractants.

Although the effects of chemokines are predominantly localised to sites of inflammation by binding to proteoglycans in the glycocalyx,${ }^{10}$ they can also be detected in the circulation, and serum concentrations of the alpha chemokine IL- 8 have been shown to correlate closely with clinical, histological, and laboratory indicators of severity in ALD. ${ }^{11-13}$ However, alcoholic hepatitis and active cirrhosis are also typically associated with a mononuclear

Abbreviations used in this paper: ALD, alcoholic liver disease; MCP, monocyte chemoattractant protein; MIP, macrophage inflammatory protein; PBMC, peripheral blood mononuclear cell; IL, interleukin; ELR, glutamine-leucine-arginine; TIPSS, transjugular intrahepatic portosystemic shunt; AST, aspartate aminotransferase. 
Table 1 Summary characteristics of patients with severe alcoholic hepatitis $(A H)$, mild to moderate AH, and quiescent cirrhosis, as defined histologically

\begin{tabular}{|c|c|c|c|}
\hline & $\begin{array}{l}\text { Severe } A H \\
(n=24)\end{array}$ & $\begin{array}{l}\text { Mild/moderate } A H \\
(n=15)\end{array}$ & $\begin{array}{l}\text { Cirrhosis } \\
(n=12)\end{array}$ \\
\hline \multicolumn{4}{|l|}{$\mathrm{AST}(\mathrm{U} / \mathrm{l})$} \\
\hline Median & 64 & 50 & 36 \\
\hline Range & $21-405$ & $20-113$ & $13-61$ \\
\hline \multicolumn{4}{|l|}{ Bilirubin $(\mu \mathrm{mol} / \mathrm{l})$} \\
\hline Median & 232 & 100 & 54 \\
\hline Range & $45-1050$ & $14-313$ & $11-550$ \\
\hline \multicolumn{4}{|l|}{$\mathrm{PT}(\mathrm{s})$} \\
\hline Median & 23 & 21 & 19 \\
\hline Range & $17-43$ & $14-39$ & $15-30$ \\
\hline \multicolumn{4}{|c|}{ Discriminant function ${ }^{\star} \dagger$} \\
\hline Median & 65.2 & 40.5 & 28.4 \\
\hline Range & $29.6-151$ & $5.6-138$ & $9.8-111$ \\
\hline PSE & 13 & 4 & 2 \\
\hline Transplanted $\ddagger$ & 2 & 3 & 3 \\
\hline Deaths $\ddagger$ & 10 & 4 & 2 \\
\hline
\end{tabular}

${ }^{\star}$ After Maddrey ${ }^{15}$ where discriminant function $=[$ (prolongation in $\mathrm{PT} \times 4.6)+($ serum bilirubin 17)]. A discriminant function of $>32$ is indicative of a risk of mortality of at least $50 \%$.

†Severe $\mathrm{AH}$ versus mild or moderate $\mathrm{AH}, \mathrm{p}<0.05$; severe $\mathrm{AH}$ versus quiescent cirrhosis, $\mathrm{p}<0.05$; mild to moderate $\mathrm{AH}$ versus quiescent cirrhosis, $\mathrm{p}<0.05$ (Mann-Whitney $\mathrm{U}$ test).

‡Occurring within three months after serum sampling. All deaths were directly related to liver disease.

AST, aspartate aminotransferase; PT, prothrombin time; PSE, portosystemic encephalopathy (defined where clinically obvious).

cell infiltrate including monocytes and lymphocytes, and the hepatic expression of the chemokines MCP-1 and MIP- $1 \alpha$ correlates with intensity of infiltration with these mononuclear cells in ALD. ${ }^{6}$ Serum concentrations of beta chemokines have not been studied in inflammatory liver diseases, although MCP-1 concentrations are elevated in human sepsis. ${ }^{14}$ For these reasons we studied circulating concentrations and peripheral secretion of MCP-1 and MIP- $1 \alpha$ in ALD in order to determine: $(a)$ whether concentrations of chemokines in the peripheral circulation reflect hepatic disease activity; and $(b)$ whether chemokine secretion is restricted to the liver or is part of a systemic inflammatory response.

\section{Patients and methods}

PATIENTS

We studied 51 patients with biopsy proved ALD. All patients had consumed at least $80 \mathrm{~g}$ alcohol daily for more than 10 years and other potential causes of liver disease had been carefully excluded by comprehensive clinical, radiological, and serological analysis. Patients were managed with nutritional supplementation but not corticosteroids or other immunosuppressants. Patients with active sepsis or recent gastrointestinal haemorrhage were excluded from the study. Patients were subdivided by histological criteria ${ }^{1}$ into those with severe alcoholic hepatitis (ballooning hepatocyte degeneration, Mallory's hyaline, and dense leucocyte infiltration including neutrophils; $\mathrm{n}=24$ ), mild to moderate alcoholic hepatitis (with ballooning hepatocyte degeneration, Mallory's hyaline, and absent or light leucocyte infiltration without neutrophils; $\mathrm{n}=15$ ), and inactive alcoholic cirrhosis (without histological stigmata of alcoholic hepatitis; $n=12$ ). The majority of patients with alcoholic hepatitis (18/24 with severe and $11 / 15$ with mild to moderate alcoholic hepatitis) also had cirrhosis, and all remaining patients had fibrosis of at least mild to moderate severity.

In addition to histological features of disease we recorded routine clinical and laboratory laboratory data for each patient (table 1). For comparison we also studied 12 healthy volunteers.

\section{SERUM SAMPLING}

Serum samples obtained from peripheral venous blood were analysed for all subjects. Some of the patients with coagulopathy underwent transjugular biopsy for clinical indications and in these patients we took the opportunity to sample blood from the hepatic vein. In a further five patients, transjugular intrahepatic portosystemic shunts (TIPSS) were inserted radiologically for control of ascites or because of previous problematic variceal haemorrhage; in these patients we took blood samples from peripheral, hepatic, and portal veins. Blood was collected into tubes containing clot activator; serum was separated within 30 minutes by centrifugation and stored at $-70^{\circ} \mathrm{C}$ prior to analysis.

PERIPHERAL BLOOD MONONUCLEAR CELL (PBMC) CHEMOKINE SECRETION

MCP-1 and MIP-1 $\alpha$ secretion from cultured PBMCs was measured in selected patients with severe alcoholic hepatitis $(n=12)$ and healthy controls $(n=12)$. PBMCs were isolated from citrated venous blood by Ficoll/Hypaque density gradient centrifugation for 30 minutes at $1600 \mathrm{rpm}$; cells were then washed twice in Hank's balanced salt solution, counted by haemocytometry, and cultured in $1 \mathrm{ml}$ aliquots at a concentration of $10^{6}$ cells $/ \mathrm{ml}$. Cells were cultured for 24 hours at $37^{\circ} \mathrm{C}$ at $10^{6}$ cells $/ \mathrm{ml}$ in RPMI containing $10 \%$ fetal bovine serum, glutamine $(2 \mathrm{mM} / \mathrm{l})$, and antibiotics (penicillin $100 \mathrm{U} / \mathrm{ml}$ and streptomycin $100 \mu \mathrm{g} / \mathrm{ml}$ ) without additional stimulation. Supernatants were collected by centrifugation and stored at $-70^{\circ} \mathrm{C}$ prior to analysis.

CHEMOKINE MEASUREMENT

MCP-1 and MIP-1 $\alpha$ concentrations were measured using commercially available ELISA kits (R\&D Systems, Abingdon, UK) according to the manufacturer's instructions. The lower limits of sensitivity for these assays were 31 and $47 \mathrm{pg} / \mathrm{ml}$ respectively. For measurement of PBMC secretion of MCP-1 we developed a customised ELISA. Ninety six microwell plates were coated with mouse monoclonal antihuman MCP-1 (500 ng/ml; R\&D Systems, Oxon, UK) overnight at $4^{\circ} \mathrm{C}$. Plates were then washed and non-specific binding blocked with $0.1 \%$ bovine serum albumin before adding the test sample for two hours at room temperature. Rabbit antihuman MCP-1 (2.5 $\mu \mathrm{g} / \mathrm{ml}$; Peprotech EC Ltd, London, UK) was added for one hour, followed by swine antirabbit antibody conjugated with horseradish peroxidase (1.3 $\mu \mathrm{g} / \mathrm{ml}$, Dako Ltd, Cambridge, UK) for a further hour, with washing between each step. Bound antibody was then detected using tetramethylene blue liquid substrate and colorimetric analysis. Reference samples consisted of serial dilutions of recombinant human MCP-1 (Peprotech EC Ltd, London, UK). This ELISA gave reproducible results with values within $10 \%$ of the commercial ELISA, with both intra-assay and interassay variability 
Figure 1 Serum monocyte chemoattractant protein 1 (MCP-1) concentrations in patients with severe or mild to moderate alcoholic hepatitis, inactive alcoholic cirrhosis, and controls. Thick horizontal bars represent mean values. Concentrations in severe alcoholic cirrhosis were significantly higher than in cirrhotics or controls ( $p<0.01$, Mann-Whitney U test).

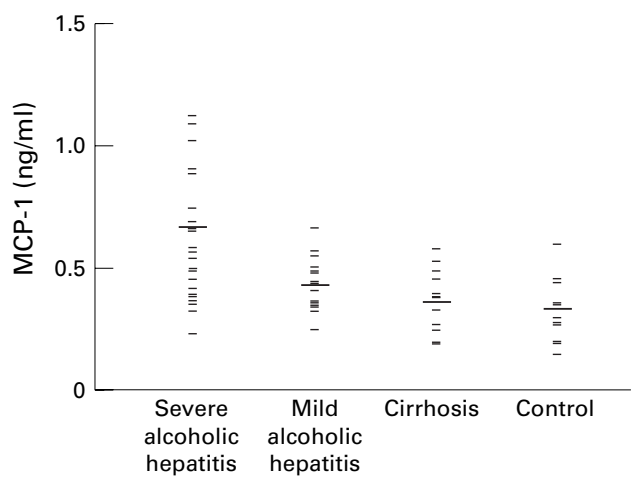

of less than $5 \%$ and with a lower limit of sensitivity of $250 \mathrm{pg} / \mathrm{ml}$.

ANALYSIS FOR PBMC CHEMOKINE MRNA

Chemokine mRNA in peripheral blood leucocytes was determined by in situ hybridisation of cytospin preparations of PBMCs isolated as described above. Freshly isolated PBMCs were resuspended and mounted onto coated slides, fixed in $1 \%$ paraformaldehyde, and stored at $-70^{\circ} \mathrm{C}$ prior to analysis. Chemokine mRNA hybridisation was done with a probe for MIP-1 $\alpha$ using techniques described elsewhere. ${ }^{4}$ Briefly, ${ }^{35} \mathrm{~S}$ uridine triphosphate labelled antisense or sense cRNA probes were synthesised from a cDNA sequence specific for MIP-1 $\alpha$, which was ligated into an Eco $\mathrm{R} 1 / \mathrm{Bam} \mathrm{H} 1$ restriction site of the plasmid vector Bluescript $\mathrm{KS}+/-$. The ${ }^{35} \mathrm{~S}$ cRNAs were characterised prior to use by polyacrylamide gel electrophoresis to ensure comparable size and specific activity. These were then hybridised with cytospin preparations and developed after 7-14 days of autoradiography.

STATISTICAL ANALYSIS

Tests for non-parametric data (Mann-Whitney U test, Wilcoxon rank sum, and multivariate linear regression) were done using SPSS statistical software. A p value of less than 0.05 was considered to indicate statistical significance. This study was approved by the Research Ethics Committee of the University Hospital Birmingham NHS Trust and patients gave informed consent before participation in the study.

\section{Results}

SERUM CHEMOKINE CONCENTRATIONS

Peripheral vein $\mathrm{MCP}-1$ concentrations were higher in alcoholic hepatitis (severe, 640 (SD 285) $\mathrm{pg} / \mathrm{ml}$; mild, 435 (110) $\mathrm{pg} / \mathrm{ml}$ ) compared

Table 2 Correlation of laboratory indexes of disease severity with serum monocyte chemoattractant protein (MCP) 1 concentrations in patients with alcoholic liver disease, using Spearman's rank test

\begin{tabular}{lll}
\hline Laboratory index & $r$ Value & p Value \\
\hline Serum aspartate aminotransferase & +0.47 & $<0.01$ \\
Creatinine $^{\star}$ & +0.36 & $<0.01$ \\
Bilirubin $^{\star}$ & +0.35 & $<0.01$ \\
Albumin & -0.33 & $<0.05$ \\
Prothrombin time increase & +0.15 & $\mathrm{NS}$ \\
Peripheral leucocyte count & +0.18 & $\mathrm{NS}$
\end{tabular}

*Values transformed logarithmically for statistical analysis. Linear regression analysis identified serum aspartate aminotransferase $(p<0.01)$ and creatinine $(p<0.05)$ as significant independent variables. with cirrhosis (355 (105) $\mathrm{pg} / \mathrm{ml}$ ) or healthy controls (325 (120) pg/ml) (severe alcoholic hepatitis versus healthy controls, $\mathrm{p}<0.01$; severe alcoholic hepatitis versus inactive cirrhosis, $\mathrm{p}<0.01$; mild alcoholic hepatitis versus healthy controls, NS; Mann-Whitney U test; fig 1). MCP-1 concentrations showed significant positive correlations with serum aspartate aminotransferase (AST) $(\mathrm{p}<0.01)$, serum creatinine $(p<0.01)$, and serum bilirubin $(\mathrm{p}<0.01)$, and a significant negative correlation with serum albumin $(\mathrm{p}<0.05)$ (table 2$)$. Linear regression analysis showed that serum AST $(p<0.01)$ and creatinine $(p<0.05)$ retained independent associations with MCP-1 concentrations. There was a non-significant trend towards higher MCP-1 concentrations in nonsurvivors compared with survivors (mean concentrations 763 and $565 \mathrm{ng} / \mathrm{ml}$ respectively, $\mathrm{p}=0.12$ ). In the alcoholic hepatitis groups, there was no difference in MCP-1 concentrations between those with and those without cirrhosis (mean concentrations 654 and 678 $\mathrm{pg} / \mathrm{ml}$ respectively, NS). Peripheral vein MIP- $1 \alpha$ concentrations were below the lower limit of detection of the assay (that is, less than $46 \mathrm{pg} / \mathrm{ml}$ ) in all healthy controls and in all ALD patients tested $(n=32)$ except one.

\section{PERIPHERAL AND HEPATIC VEIN CHEMOKINE} CONCENTRATIONS

In patients with severe alcoholic hepatitis in whom paired hepatic and peripheral vein samples were available, MCP-1 concentrations were higher in hepatic compared with peripheral venous blood (mean values 647 and 600 $\mathrm{pg} / \mathrm{ml}$ respectively, $\mathrm{p}<0.01$, Wilcoxon rank sum), while in patients with mild to moderate alcoholic hepatitis or inactive cirrhosis the hepatic and peripheral vein MCP-1 concentrations were similar (mean values 293 and 301 $\mathrm{pg} / \mathrm{ml}$ respectively, NS; fig 2). In the five patients undergoing TIPSS insertion, the highest MCP-1 concentrations were found in the hepatic veins, with the lowest concentrations occurring in the portal veins (mean concentrations in the hepatic, peripheral, and portal veins were 437, 421, and $402 \mathrm{pg} / \mathrm{ml}$ respectively; hepatic vein versus portal vein, $p=0.07$, Wilcoxon rank sum). Hepatic vein MIP-1 $\alpha$ concentrations were below the lower limit of

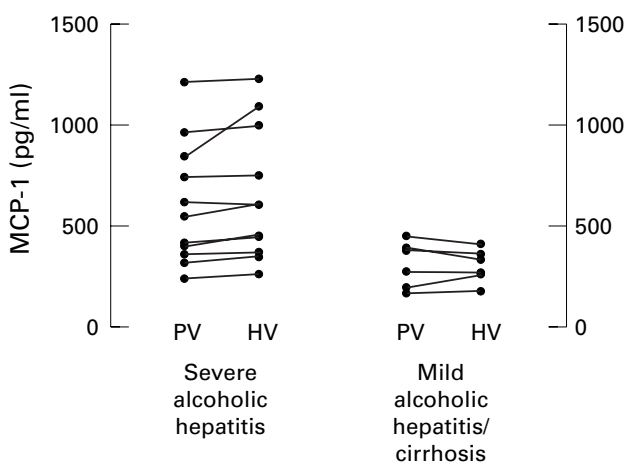

Figure 2 Paired hepatic and peripheral vein (HV and $P V)$ monocyte chemoattractant protein 1 (MCP-1) concentrations in patients with severe alcoholic hepatitis compared with mild to moderate hepatitis or cirrhosis. 
Figure 3 Spontaneous peripheral blood mononuclear cell secretion of $(A)$ monocyte chemoattractant protein 1 $(M C P-1)$ and $(B)$ macrophage inflammatory protein $1 a(M I P-1 a)$ in patients with severe alcoholic hepatitis and healthy controls. Thick horizontal bars represent mean values.
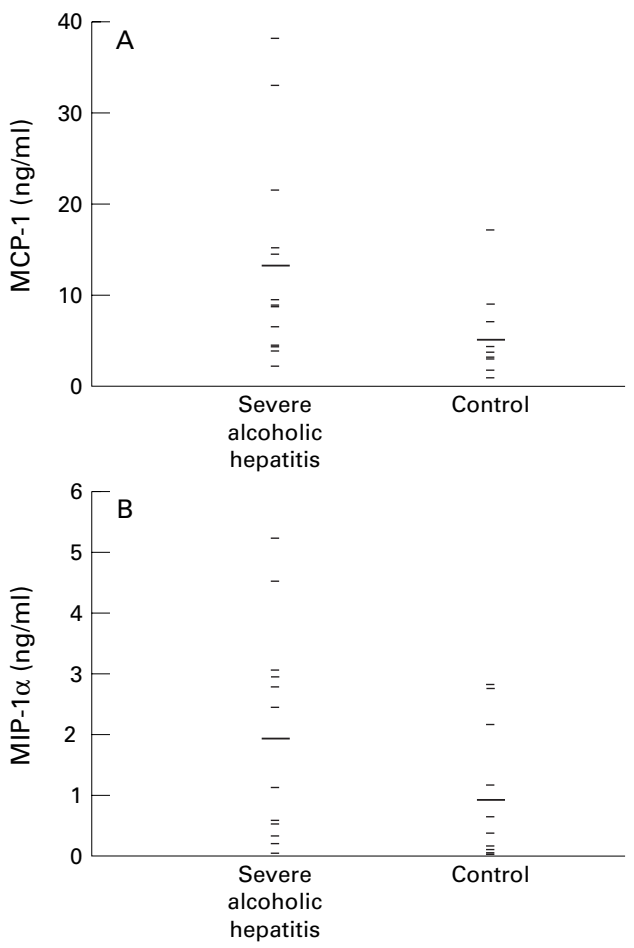

detection of the assay (as for peripheral vein concentrations) in all patients with ALD.

PBMC CHEMOKINE SECRETION

Spontaneous secretion of MCP-1 and MIP- $1 \alpha$ from cultured PBMCs was higher in patients with severe alcoholic hepatitis compared with healthy controls (fig 3). In alcoholic hepatitis MCP-1 secretion was $13.13(11.34) \mathrm{ng} / \mathrm{ml}$ and for controls 5.27 (4.77) $\mathrm{ng} / \mathrm{ml} \quad(\mathrm{p}=0.015$, Mann-Whitney U test); MIP-1 $\alpha$ secretion was $1.98(1.78) \mathrm{ng} / \mathrm{ml}$ in alcoholic hepatitis and $0.84(1.10) \mathrm{ng} / \mathrm{ml}$ in controls ( $\mathrm{p}=0.04$, MannWhitney U test). For patients with alcoholic hepatitis there was a significant positive correlation between PBMC secretion of and serum concentrations of MCP-1 $(r=0.68, \mathrm{p}<0.05$, Spearman's rank test), and between PBMC secretion of MCP-1 and serum AST $(r=0.60$, $\mathrm{p}<0.05)$. However, there was no significant correlation between PBMC secretion of MIP-1 $\alpha$ with MCP-1 secretion or serum AST.

IDENTIFICATION OF CHEMOKINE mRNA

In situ hybridisation of cytospin preparations using the probe for MIP-1 $\alpha$ mRNA showed positive staining (mainly localised to monocytes) in patients with alcoholic hepatitis (fig 4).

\section{Discussion}

Our results suggest that secretion of MCP-1 is associated with disease activity in ALD. These findings complement previous observations of chemokine upregulation in the liver in ALD and suggest a role for chemokines in recruiting mononuclear cells to the liver following alcohol induced toxic injury. Previous reports of circulating IL-8 in alcoholic hepatitis have highlighted the importance of neutrophils in the pathogenesis of alcoholic hepatitis ${ }^{11-13}$; however, while a neutrophilic infiltrate is the char-

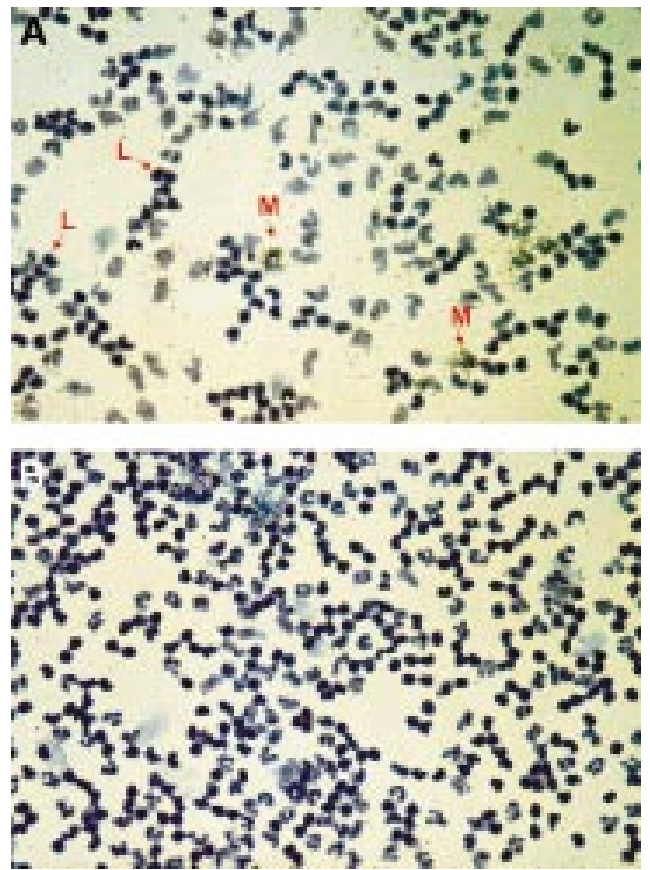

Figure 4 PBMC cytospin preparation hybridised with $c D N A$ for macrophage inflammatory protein 1a (MIP-1a) chemokine $m R N A$ ( $A$, antisense probe; $B$, sense probe).

Positive hybridisation is represented by dark granules, which are seen mostly in association with monocytes (M); lymphocytes $(L)$ are mostly negative.

acteristic hallmark of alcoholic hepatitis, the inflammatory infiltrate includes mononuclear cells which play a vital role in inflammation and fibrogenesis. ${ }^{17}$ Thus beta chemokines such as MCP-1 are likely to play a central role in mediating these processes in ALD. We were unable to show increased serum concentrations of the beta chemokine MIP- $1 \alpha$ in ALD despite showing enhanced PBMC secretion; it is possible that circulating concentrations were increased but below the range detectable by the assay.

The significance of raised circulating concentrations of MCP-1 remains unclear and while this may simply reflect increased tissue synthesis of MCP-1, circulating MCP-1 may also have immunomodulatory effects, including increased expression of adhesion molecules on circulating monocytes and promotion of proinflammatory cytokine secretion, thus amplifying the inflammatory cascade. ${ }^{16}$ Furthermore, circulating IL- 8 has been shown to enhance neutrophil sequestration into the liver and MCP-1 may play a similar role in enhancing hepatic uptake of mononuclear cells. ${ }^{17}$ However, the dynamic effects of a sustained increase in circulating chemokines are not clear and it is possible that persistent ligand occupancy of leucocyte chemokine receptors from circulating chemokines may inhibit their transendothelial migration. ${ }^{18}$

Our finding of a positive correlation between MCP-1 concentrations and serum creatinine probably reflects in part the renal excretion of chemokines; by virtue of its relatively small protein size, some excretion of MCP-1 is likely to occur from the kidneys. Measurable amounts are present in the urine of most healthy subjects ${ }^{19}$ and following this study we found high concentrations (1.34 and $1.36 \mathrm{ng} / \mathrm{ml}$ respec- 
tively) in the urine of two patients with alcoholic hepatitis (but without renal failure), corresponding to at least twice the upper limit of normal renal values. Renal failure is therefore likely to increase circulating MCP-1 concentrations by a reduction in urinary excretion; however, in addition renal failure itself may promote synthesis of MCP-1 and other cytokines, including from intrarenal sources. ${ }^{2021}$

Increased secretion of MCP-1 from circulating monocytes, and increased MCP-1 concentrations in hepatic compared with peripheral (and portal) veins, suggest that MCP-1 synthesis is increased both peripherally and within the liver, respectively, in severe alcoholic hepatitis. While we found hepatic vein concentrations to be only modestly higher than peripheral concentrations, this difference was nevertheless significant and is remarkable in that patients were sampled in a "steady state" in the absence of any hepatic insult other than the ongoing inflammatory process. Thus, the increase in hepatic vein concentrations suggests synthesis within the liver.

Our observations support a direct role for MCP-1 in disease pathogenesis, presumably by recruiting and retaining monocytes and activated lymphocytes within the liver in severe alcoholic hepatitis. The fact that circulating monocytes showed increased spontaneous secretion of MCP-1 in alcoholic hepatitis suggests that recruitment of these cells into the liver would further elevate local concentrations and act as an amplifying signal to enhance liver inflammation. The circulating monocytes might have been activated within the hepatic circulation during transit through the hepatic sinusoids or directly by soluble circulating factors.

The stimulus for chemokine production in ALD is not known but is likely to involve several signals. Proinflammatory cytokines (such as tumour necrosis factor $\alpha(\mathrm{TNF}-\alpha)$ and IL-1) and lipopolysaccharide are potent inducers of MCP-1; all these factors have been reported to be increased in the circulation in alcoholic hepatitis. ${ }^{22}{ }^{23}$ The stimulus within the liver might be local TNF- $\alpha$, the expression of which is increased in severe alcoholic hepatitis, ${ }^{64}$ or the direct effects of alcohol metabolites or alcohol induced reactive oxygen mediators. Synthesis of the chemokines is likely to occur predominantly from Kupffer and stellate cells within the liver. ${ }^{325}$ Alcohol itself is unlikely to act as a direct stimulus as this normally decreases cytokine or chemokine secretion $^{26}$; however, toxic alcohol metabolites may induce a "rebound" increase in cytokine synthesis following withdrawal of alcohol. ${ }^{12} 27$

The further evidence we provide of a role for chemokines in the pathogenesis of ALD has therapeutic implications, particularly in light of observations that mortality in severe alcoholic hepatitis remains high even after withdrawal of alcohol. ${ }^{28}$ If, as we propose, MCP-1 plays a central role in this process then treatment aimed at preventing MCP-1 secretion or inhibiting its effects might present an opportunity to alter the clinical course of an otherwise devastating disease.
We are grateful to Dr Simon Olliff for help in obtaining hepatic and portal venous blood samples from patients undergoing radiological procedures. We are also grateful to our physician colleagues for allowing us to study patients under their care and to Dr Clare Morland and Janine Fear for practical help. This work was supported in part by grants from the Sir Jules Thorn Trust and the Endowment Fund of the former United Birmingham Hospitals.

1 Anonymous. Alcoholic liver disease: morphological manifestations. Lancet 1981;i:707-11.

2 Paronetto F. Immunological reactions in alcoholic liver disease. Semin Liver Dis 1993;13:183-95.

3 Czaja MJ, Geerts A, Xu J, et al. Monocyte chemoattractant protein 1 (MCP-1) expression occurs in toxic rat liver injury and human liver disease. $\mathcal{F}$ Leukocyte Biol 1994;55: $120-6$.

4 Adams DH, Afford SC, Fear J, et al. Hepatic expression of macrophage inflammatory protein- $1 \beta$ after liver transplantation. Transplantation 1996;61:817-25.

5 Maltby J, Wright S, Bird G, et al. Chemokine levels in human liver homogenates: association between GRO alpha and histopathological evidence of alcoholic hepatitis. Hepatology 1996;24:1156-60.

6 Afford SC, Fisher NC, Neil D, et al. Distinct patterns of chemokine expression are associated with leucocyte recruitment in alcoholic hepatitis and alcoholic cirrhosis. $\mathcal{F}$ Pathol 1998;186:82-9.

7 Marra F, DeFranco R, Grappone C, et al. Increased expression of monocyte chemotactic protein-1 during active hepatic fibrogenesis-correlation with monocyte infiltration. Am f Pathol 1998;152:423-30.

8 Baggiolini $M$. Chemokines and leukocyte traffic. Nature 1998;392:565-8.

9 Luster AD. Chemokines-chemotactic cytokines that mediate inflammation. $N$ Engl f Med 1998;338:436-45.

10 Tanaka Y, Kimata K, Adams DH, et al. Modulation of cytokine function by heparan sulfate proteoglycans: sophisticated models for the regulation of cellular responses to cated models for the regulation of cellular resp
cytokines. Proc Ass Am Phys 1998;110:118-25.

11 Hill DB, Marsano LS, McClain CJ. Increased plasma interleukin-8 concentrations in alcoholic hepatitis. Hepatology 1993;18:576-80.

12 Masumoto $\mathrm{T}$, Onji $\mathrm{M}$, Horiike $\mathrm{N}$, et al. Assay of serum interleukin-8 levels in patients with alcoholic hepatitis. Alcohol Alcohol 1993;28:99-102.

13 Sheron N, Bird G, Koskinas J, et al. Circulating and tissue levels of the neutrophil chemotaxin interleukin-8 are elevated in severe acute alcoholic hepatitis, and tissue levels correlate with neutrophil infiltration. Hepatology 1993;18: 41-6.

14 Bossink AW, Paemen L, Jansen PM, et al. Plasma levels of the chemokines monocyte chemotactic proteins- 1 and -2 are elevated in human sepsis. Blood 1995;86:3841-7.

15 Maddrey WC. Alcoholic hepatitis: pathogenesis and approaches to treatment. Scand f Gastroenterol 1990;175:11830 .

16 Jiang Y, Beller DI, Frendl G, et al. Monocyte chemoattractant protein-1 regulates adhesion molecule expression and cytokine production in human monocytes. F Immunol 1992;148:2423-8.

17 Van Zee KJ, Fischer E, Hawes AS, et al. Effects of intravenous IL-8 administration in nonhuman primates. $\mathcal{F}$ Immunol 1992;148:1746-52.

18 Luscinskas FW, Kiely JM. Ding H, et al. In vitro inhibitory effect of IL-8 and other chemoattractants on neutrophilendothelial adhesive interactions. F Immunol 1992;149: 2163-71.

19 R\&D Quantikine (Human MCP-1 immunoassay) handbook. Catalogue no. DCP00. Oxon, UK: R\&D Systems Europe.

20 Abdullah MS, Wild G, Jacob V, et al. Cytokines and the malnutrition of chronic renal failure. Miner Energy Metab 1997;23:237-42.

21 Akahoshi T, Kobayashi N, Hosaka S, et al. In-vivo induction of monocyte chemotactic and activating factor in patients with chronic renal failure. Nephrol Dial Transplant 1995;10: $2244-9$

22 Khoruts A, Stahnke L, McClain CJ, et al. Circulating tumor necrosis factor, interleukin- 1 and interleukin- 6 concentrations in chronic alcoholic patients. Hepatology 1991;13: 267-76

23 Bode C, Kugler V, Bode JC. Endotoxaemia in patients with alcoholic and non-alcoholic cirrhosis and in subjects with no evidence of chronic liver disease following acute alcohol excess. F Hepatol 1987;4:8-14.

24 McClain CH, Schmidt J, Diehl AM. Cytokines and alcoholic liver disease. Semin Liver Dis 1993;13:170-82.

25 Armendariz-Borunda J, Seyer JM, Postlethwaite AE, et al. Kupffer cells from carbon tetrachloride-injured rat liver produce chemotactic factors for fibroblasts and monocytes: produce chemotactic factors for fibroblasts and monocytes:
the role of tumour necrosis factor-alpha. Hepatology 1991; 14:895-900.

26 Maher JJ. Rat hepatocytes and Kupffer cells interact to produce interleukin-8 (CINC) in the setting of alcohol. Am f Physiol 1995;269: G518-23.

27 Enomoto N, Ikejima K, Bradford B, et al. Alcohol causes both tolerance and sensitization of rat Kupffer cells via mechanisms dependent on endotoxin. Gastroenterology 1998;115:443-51

28 Orrego H, Blake JE, Blendis LM, et al. Prognosis of alcoholic cirrhosis in the presence and absence of alcoholic hepatitis. Gastroenterology 1987;92:208-14. 